
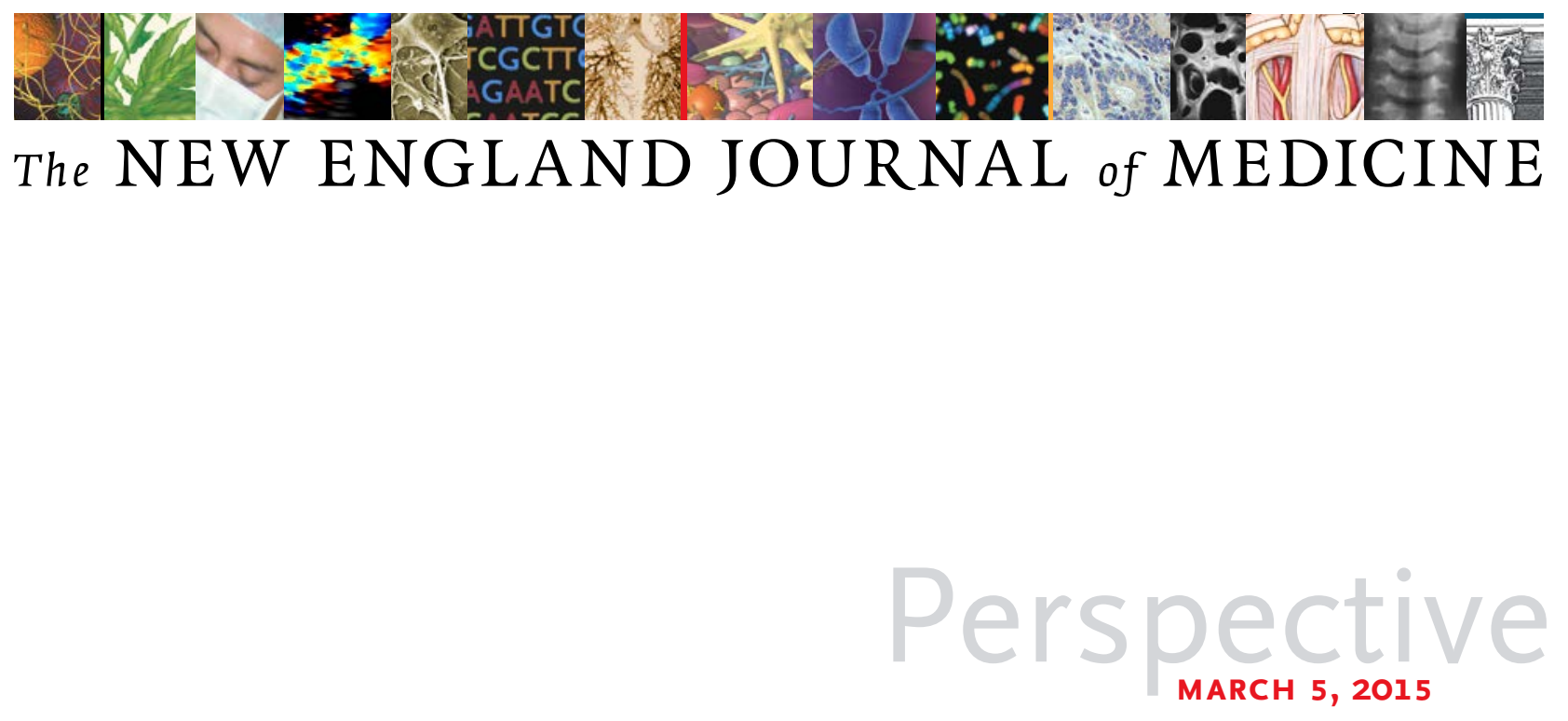

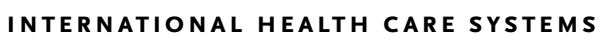

\title{
A Precious Jewel - The Role of General Practice in the English NHS
}

Martin Marshall, M.D.

\section{Eneral practice (or family medicine, as it is $\checkmark$ known in the United States) is often described as "the jewel in the crown" of the English National Health Service (NHS). It contributes to the high}

ranking of the English health system (see table and case histories) in international comparisons in three ways. ${ }^{1}$ At the level of individual patients, it provides care for people presenting with most common conditions, with a growing emphasis on patients with multiple coexisting conditions, and a commitment to continuity and An interactive $\mid$ whole-person care. graphic is At a population levavailable at NEJM.org el, general practice serves defined local communities, so it can focus on prevention and health education as well as the treatment of disease. And at a health-system level, general practice delivers efficient care by managing clinical risk through its front-line services, preventing overmedicalization, facilitating appropriate access to specialist services and investigations, and advocating for patients when necessary.

The combination of these central roles and a remarkably high level of public support and trust has, in the past, caused general practice to be taken for granted by policymakers, who have generally focused on the more politically charged hospital sector. Sporadic experiments over recent decades with innovative pay-for-performance schemes and a range of models under which primary care-led groups assume respon- sibility for purchasing (commissioning) hospital and community services for a given population cannot hide the reality that policymakers have failed to engage either health care professionals or the public with a coherent, comprehensive vision for general practice.

But the political and media spotlight is now being turned on this sector. General practice is being criticized for its small scale, isolation, and lack of accountability. Structures that used to be praised as entrepreneurial and flexible are now described pejoratively as a "cottage industry." Unacceptable performance in a small number of practices is being highlighted rather than ignored. But most important, this new focus reflects a growing concern that the majority of general practices have neither the 
Selected Characteristics of the Health Care System and Health Outcomes in England.*

\section{Variable}

Health expenditures

Per capita (U.S. \$)

Percentage of GDP

Public sources (\% of total)

Health insurance

Rate in population (\%)

Source of funding

Physician income (U.S. \$)

Salaried general practitioner in 2011

Self-employed general practitioner in 2011

Salaried specialist in 2013

Generalist-specialist balance in 2013 (\%)

Generalists

Specialists

Access

No. of hospital beds per 10,000 population in 2011

No. of physicians per 1000 population

Primary care physicians using electronic medical records (\%)

2.8

97

Life and death

Life expectancy at birth (yr)

82

Additional years of life expectancy at $60 \mathrm{yr}$

24

Annual no. of deaths per 1000 population

Annual no. of infant deaths per 1000 live births in 2013

Annual no. of deaths of children $<5$ yr of age per 1000 live births in 2013

Annual no. of maternal deaths per 100,000 live births in 2013

Fertility: average no. of births per woman

Preventive care

General availability of colorectal-cancer screening at primary care level

Children 12-23 mo of age receiving measles immunization in 2013 (\%)

Not

95

Prevalence of chronic disease

Diabetes (\% of 2013 population $20-79 \mathrm{yr}$ of age)

4.9

HIV infection

Prevalence of risk factors (\%)

Obesity in adults $\geq 18 \mathrm{yr}$ of age in 2014』

Smoking in 2011 in persons $>16 \mathrm{yr}$ of age

21.0

* Data are from the World Bank, the Organization for Economic Cooperation and Development, the Commonwealth Fund, and the World Health Organization and are for 2012 except as noted. GDP denotes gross domestic product, and HIV human immunodeficiency virus.

$\dagger$ This figure represents income before practice expenses are deducted. The average National Health Service income for the main contractual services is $£ 102,000$ (approximately U.S. $\$ 156,500$ ), but that does not include income from other sources.

7 Colorectal-cancer screening is available for high-risk groups.

$\int$ Obesity in adults was defined as a body-mass index (the weight in kilograms divided by the square of the height in meters) of 30 or more. 
capacity nor the capability to respond to the increasing expectations placed on them by the public and policymakers - expectations that they will be able to cope with both the increase in the consultation (visit) rate from 3.9 consultations per patient per year in 1995 to 5.5 in $2009^{2}$ and the increasing complexity of most of these consultations; that they will assume responsibility for the growing number of activities that are being shifted out of hospitals and into the community; that they will adopt the lead role in commissioning health services, with a more proactive, integrated, and population-based orientation; and that they will be at the forefront of technological and social innovation in care delivery.

These growing expectations are hitting general practice at a difficult time. Overall spending on the NHS has risen by $18 \%$ since the 2005-2006 fiscal year, but the proportion of the budget allocated to general practice has dropped by $8 \%$ over the same period. ${ }^{3}$ In the past year, there has been a $15 \%$ decrease in applications for general practice training posts ${ }^{4}$; practices are finding it increasingly difficult to fill vacant equity-holding partnerships and salaried posts, and they are struggling to hold onto the growing number of older doctors who are considering early retirement. A specialty that has historically had an admirable reputation for simply absorbing whatever it was asked to do is now struggling to deal with the growing expectations that are being placed on it. Some commentators are saying that general practice is in crisis.

There are some encouraging signs that NHS leaders recognize that they need to take urgent ac-

\section{MYOCARDIAL INFARCTION}

A 55-year-old man with no serious health conditions has a moderately severe myocardial infarction.

The management pathway for people with a suspected myocardial infarction is well established in the United Kingdom. When Mr. Taylor has chest pain and tightness one day at work and breaks out into a cold sweat, his secretary calls an ambulance. The paramedics obtain an electrocardiogram, and since it shows evidence of an infarction, Mr. Taylor is rapidly taken to an on-call interventional cardiac unit for primary treatment with percutaneous coronary intervention. "Call-to-balloon" times are closely monitored nationally, with a target of 90 minutes which is achieved in Mr. Taylor's case, as it is in most others. If he had not been eligible for this treatment or did not live near an appropriate facility, as some people do not, thrombolytic agents would be administered, if they weren't contraindicated, again with a target "door-to-needle" time of 90 minutes.

Mr. Taylor is closely monitored on the cardiac unit, and blood is drawn for laboratory testing to assess his progress, any complications, and risk factors. After treatment for the acute problem, he is transferred to a step-down cardiac ward, and if he has no complications he can be discharged home within 3 to 4 days after admission.

Four to 6 weeks after discharge, Mr. Taylor's health will be reviewed in a cardiology outpatient office by a consultant or an interventional nurse specialist, who will assess him for complications and ensure that all his cardiovascular risks are appropriately managed. Mr. Taylor's general practitioner is then responsible for providing ongoing holistic care, including managing risks and monitoring routinely prescribed antiplatelet therapy, beta-blockers, statins, and angiotensin-converting-enzyme inhibitors. Cardiac rehabilitation should start within 10 days after discharge, though implementation of that recommendation varies, in part because of limitations in service provision and in part owing to patient choice.

tion. The newly appointed chief executive officer of NHS England (who spent several years as a senior executive officer at UnitedHealth in the United States) has set out a vision for the health service ${ }^{5}$ that demonstrates a clear commitment to strengthening the role of primary care. Policymakers plan to invest new funding in general practice, to break down the barriers between primary and secondary care, and to shift resources from the hospital sector into the community. Workforce planners are recognizing the need to attract a higher proportion of trainee doctors and nurses into general practice. They are start- 


\section{PREGNANCY AND CHILDBIRTH}

\section{A healthy 23-year-old woman is pregnant for the first time.}

Maternity care in the United Kingdom is freely available from the National Health Service for all women, and national guidance outlines the nature of the care that should be offered, strongly emphasizing women's choice. When Ms. Williams becomes pregnant, she contacts her general practitioner (GP), who provides her with routine information and assesses her risk factors, advising her to take folic acid and, if she is considered to be at high risk, vitamin D. She is then booked for "shared care" between a midwife (midwives are responsible for the delivery of most of the antenatal care in community settings) and a hospital-based obstetrician. Apart from the initial contact, Ms. Williams's GP is less involved than he or she might have been in the past, though some GPs share antenatal care with midwives and remain responsible for routine general medical care throughout a patient's pregnancy. Ms. Williams owns and carries a hard copy of her maternity records, and the data are duplicated in NHS electronic records.

Ms. Williams's first appointment with a midwife takes place at 8 to 12 weeks of gestation. She is given comprehensive information about antenatal and intrapartum care, and she undergoes screening blood tests for anemia, blood group, $\mathrm{Rh}$ antibodies, sickle cell disease, thalassemia, diabetes, and relevant infections. She is also offered screening for Down's syndrome. Since she has no complications, she undergoes two ultrasonographic scans, one at 12 weeks for dating and one between 18 and 21 weeks for anomalies.

Ms. Williams is seen in a community-based antenatal clinic on approximately 10 occasions throughout her pregnancy for routine monitoring, going more frequently after 24 weeks of gestation. Since this is her first baby, she would until recently have been advised to give birth in the hospital, usually in a midwifeoperated unit but with obstetrical expertise readily available. Recent national guidance has highlighted that home births carry no greater risks than hospital-based births for low-risk mothers.

ing to implement radical changes in undergraduate training, with more time spent learning in community settings and an improved career structure and working environment to encourage the brightest students to opt for a career in the community and to retain the physicians who are already there.

But the problem with finan- cial and workforce solutions is that they are difficult to implement and slow to deliver. In the meantime, the most visible manifestation of reform in general practice is the growing number of initiatives that are shaking up long-established structures and governance arrangements. The relatively standardized historical model of general practice that was lauded in the recent past is being replaced by a heterogeneous mix of innovative employment contracts, practice mergers, newly established networks, forprofit providers, and plans for vertically integrating services by encouraging acute care hospitals to take over struggling practices.

What is emerging is, in essence, a complex mix of forms and governance arrangements similar to that seen in the United States. Any suggestion that England might copy or learn from a specialist-dominated health system will concern the many people who admire the established model of general practice. But in some ways, the changes make sense: hard times demand a willingness to think and act differently. In the absence of sound evidence regarding what works, and given the urgent need for solutions to the impending crisis, experimentation with new models is justified, as long as they are implemented with a strong commitment to learning from contemporaneous rigorous evaluation.

Many observers will be skeptical: the NHS has a long history of reorganizing structures while ignoring cultures and working practices, and a growing body of international research suggests that structural change rarely offers an effective or sustained solution to problems with the quality of care. The real challenges are to focus on maintaining and building the principles that have made general practice successful and to ensure that these principles underpin new models of care. I believe that whatever new models are followed, experienced generalist clinicians should remain on the front line of care, meeting the health needs of most 
people most of the time and managing access to specialist services. To do so, they will have to get the support they need to continue to provide person-centered care and to deal with the complex and delicate balance among an individual's health, illness (the perception that something is wrong), and disease (a confirmed diagnosis). General practices will have to facilitate the increasingly important interface between people's management of their own health and the care that is delivered in partnership with, or by, health care professionals. And they will have to find ways to negotiate the complex trade-offs among the sometimes conflicting expectations and needs of individuals, populations, and taxpayers, whose continuing support for a publicly funded health system is essential for its survival.

If these principles underpin future models of general practice, then it matters little how the care is structured or who is responsible for managing it. In 10 years' time, general practice in England may look very different from how it looks today, but it would be a disaster if the assets that general practice has historically brought to the NHS are carelessly lost in the name of reform.

Disclosure forms provided by the author are available with the full text of this article at NEJM.org.
From the Department of Primary Care and Population Health, University College London, London.

1. Commonwealth Fund. Mirror, mirror on the wall, 2014 update: how the US healthcare system compares internationally. 2014 (http:// www.commonwealthfund.org/publications/ fund-reports/2014/jun/mirror-mirror).

2. Health and Social Care Information Centre. Trends in GP consultation rates 1995-2009. 2009 (http://www.hscic.gov.uk/ pubs/gpcons95-09).

3. Royal College of General Practitioners. Put patients first. 2014 (http://www.rcgp.org .uk/policy/put-patients-first.aspx).

4. Health Education England. Securing the future GP workforce: delivering the mandate on GP expansion. GP Taskforce final report. 2014 (http://hee.nhs.uk/wpcontent/uploads/ sites/321/2014/07/GP-Taskforce-report.pdf). 5. England NHS. Five year forward view. 2014 (http://www.england.nhs.uk/wp-content/ uploads/2014/10/5yfv-web.pdf).

DOI: 10.1056/NEJMp1411429

Copyright (c) 2015 Massachusetts Medical Society.

\section{Setting Value-Based Payment Goals - HHS Efforts to Improve U.S. Health Care}

Sylvia M. Burwell

Tow that the Affordable Care Act (ACA) has expanded health care coverage and made it affordable to many more Americans, we have the opportunity to shape the way care is delivered and improve the quality of care systemwide, while helping to reduce the growth of health care costs. Many efforts have already been initiated on these fronts, leveraging the ACA's new tools. The Department of Health and Human Services (HHS) now intends to focus its energies on augmenting reform in three important and interdependent ways: using incentives to motivate higher-value care, by increasingly tying payment to value through alternative payment models; changing the way care is delivered through greater teamwork and integration, more effective coordination of providers across settings, and greater attention by providers to population health; and harnessing the power of information to improve care for patients.

As we work to build a health care system that delivers better care, that is smarter about how dollars are spent, and that makes people healthier, we are identifying metrics for managing and tracking our progress. A majority of Medicare fee-for-service payments already have a link to quality or value. Our goal is to have $85 \%$ of all Medicare fee-forservice payments tied to quality or value by 2016 , and $90 \%$ by 2018. Perhaps even more impor- tant, our target is to have $30 \%$ of Medicare payments tied to quality or value through alternative payment models by the end of 2016, and $50 \%$ of payments by the end of 2018. Alternative payment models include accountable care organizations (ACOs) and bundled-payment arrangements under which health care providers are accountable for the quality and cost of the care they deliver to patients. This is the first time in the history of the program that explicit goals for alternative payment models and value-based payments have been set for Medicare. Changes assessed by these metrics will mark our progress in the near term, and we are engaging state Medicaid programs and private payers in efforts to 